\title{
Electrocardiographic Associations with Myocardial Fibrosis Among Sudden Cardiac Death Victims
}

Lauri T.A. Holmström ${ }^{\mathrm{a}}$, BM; M. Anette Haukilahti ${ }^{\mathrm{a}}$, BM; Juha Vähätalo ${ }^{\mathrm{a}}$, BM; Tuomas V. Kenttäa ${ }^{\mathrm{a}}$ PhD; Henrik Appel $^{\mathrm{a}}$, BM; Antti Kiviniemi ${ }^{\mathrm{a}}, \mathrm{PhD}$; Lasse Pakanen ${ }^{\mathrm{b}, \mathrm{c}}, \mathrm{MD}, \mathrm{PhD}$; Heikki V. Huikuri ${ }^{\mathrm{a}}, \mathrm{MD}, \mathrm{PhD}$; Robert J. Myerburgd. MD, PhD; M. Juhani Junttila ${ }^{\mathrm{a}}$, MD, PhD.

Author Affiliations:

\author{
${ }^{a}$ Research Unit of Internal Medicine, Medical Research Center Oulu, University of Oulu and \\ Oulu University Hospital, Oulu, Finland. \\ ${ }^{\mathrm{b}}$ Forensic Medicine Unit, National Institute for Health and Welfare, Oulu, Finland \\ ${ }^{\mathrm{c}}$ Department of Forensic Medicine, Research Unit of Internal Medicine, Medical Research Center \\ Oulu, University of Oulu, Oulu, Finland \\ ${ }^{\mathrm{d}}$ Division of Cardiology, Miller School of Medicine, University of Miami, Miami, FL
}

Disclosures: No relevant conflicts of interest related with the topic

Word Count: 3185

Running head: Myocardial fibrosis and ECG

Key Words: Electrocardiography, Sudden Cardiac Death, Fibrosis

Corresponding author: Lauri Holmström, BM, Medical Research Center Oulu, Oulu University Hospital and University of Oulu, PO Box 5000, Oulu, FIN-90014, Finland. E-mail: lauri.holmstrom@student.oulu.fi. Phone: +358 8 3154464. Fax \#: +358-8-315 5599. 


\section{ABSTRACT}

Objective. A major challenge in reducing the incidence of sudden cardiac death (SCD) is the identification of patients at risk. Myocardial fibrosis has a substantial association with SCD risk, but is difficult to identify among general populations. Our aim was to find electrocardiographic (ECG) markers of myocardial fibrosis among SCD victims.

Methods. Study population was acquired from the Fingesture study, which has gathered data from 5,869 consecutive autopsied SCD victims in Northern Finland between 1998 and 2017. The degree of fibrosis was determined in histological samples taken from the heart during autopsy and was categorized into four groups; 1) no fibrosis, 2) scattered mild fibrosis, 3) moderate patchy fibrosis and 4) substantial fibrosis. We were able to collect ECGs from 1,100 SCD victims.

Results. The mean age of the study subjects was $66 \pm 13$ years and $75 \%$ were male. QRS duration in ECG correlated with the degree of fibrosis $(\mathrm{p}<0.001, \beta=0.153)$. Prevalence of fragmented QRS complex, pathologic $\mathrm{Q}$ waves, and T-wave inversions correlated with increased degree of fibrosis $(\mathrm{p}<0.001$ in each). Depolarization abnormalities were observed both in ischemic and non-ischemic heart disease. Repolarization abnormalities reached statistical significance only among ischemic SCD victims. An abnormal ECG was observed in $75.3 \%$ of the subjects in group 1,73.7\% in group 2, $88.5 \%$ in group 3 and $91.7 \%$ in group 4 patients $(\mathrm{p}<0.001)$.

Conclusions. Myocardial fibrosis was associated with QRS prolongation, deep Q waves, T-wave inversions and QRS fragmentation. The results provide potentially useful non-invasive early recognition of patients with fibrotic cardiomyopathy and risk of SCD. 


\section{KEY QUESTIONS}

What is already known about this subject?

- Sudden cardiac death often occurs due to undiagnosed and untreated structural cardiac disease with excessive myocardial fibrosis.

What does this study add?

- Various depolarization and repolarization abnormalities in 12-lead ECG correlated with the amount of myocardial fibrosis among sudden cardiac death victims.

How might this impact on clinical practice?

- Patients with such ECG abnormalities in the absence of structural cardiac disease may require further investigations for diagnosis and classification, which may affect the risk for SCD. 


\section{INTRODUCTION}

Despite the progress in prevention and treatment of cardiac diseases, the overall incidence of sudden cardiac death $(\mathrm{SCD})$ has not declined during the last decades ${ }^{1}$. Ischemic heart disease is the most common cause of $\mathrm{SCD}^{2}$ but $25 \%$ of SCD victims have a non-ischemic cause ${ }^{1,3}$. Although ischemic and nonischemic heart disease arise from divergent pathophysiologies, expression of cardiac hypertrophy with myocardial fibrosis appears to be a common denominator in both entities. Magnetic resonance imaging (MRI) is commonly used in clinical practice for detection of myocardial fibrosis, but it is not feasible for large scale population screening.

As more than $70 \%$ of SCDs occur in patients who do not fulfill the criteria for prophylactic device

therapy, ${ }^{4}$ the need for novel risk markers that classify individual risk is significant ${ }^{5}$. The standard 12-lead electrocardiogram (ECG) has attained attention in this regard and many depolarization and repolarization abnormalities have been found to associate increased SCD risk in various populations ${ }^{6}$. However, such ECG abnormalities have not yet proven to be useful enough in SCD risk stratification to have effect on clinical decision-making. In addition, the usefulness of ECG risk markers is not limited to SCD risk stratification, but also for early recognition of subjects with underlying life-threatening heart disease generally. The potential value is suggested by the fact that up to $50 \%$ of all SCD victims do not have a prior diagnosis of cardiac disease before SCD. The percentage is even higher among those with nonischemic SCDs ${ }^{3}$.

We studied the association between ECG abnormalities and the degree of myocardial fibrosis among autopsy-verified SCD victims in order to identify the presence of life-threatening fibrotic heart disease. 


\section{METHODS}

\section{Study population}

Our study population is derived from the Fingesture study, which includes autopsy data from 5,869 consecutive SCD victims from Northern Finland and Lapland during 1998-2017. In all cases, a medicolegal autopsy was performed in Department of Forensic Pathology, University of Oulu and National Institute of Health and Welfare, Oulu, Finland. Autopsies were performed by experienced forensic pathologists using contemporary guidelines for the diagnosis of the cause of death. Finnish law requires medicolegal autopsy to be performed if the death is not caused by a known disease, the victim has not been treated during his/her last illness by physician, the death if from non-natural causes, or the death is otherwise unexpected. Consequently, Finland has the highest sudden death autopsy rate in Western societies ${ }^{7}$.

In this autopsy-based study, sudden death was defined as witnessed death within 6 hours of the onset of the symptoms and as unwitnessed death within 24 hours of the time the victim was last seen in a stable state of health. Subjects in whom autopsy revealed non-cardiac causes of sudden death, e.g. pulmonary embolism, cerebral hemorrhage or non-natural causes, were not included in the Fingesture study. Hence, the Fingesture study includes only the sudden death victims with proven cardiac origin (specifically, $\mathrm{SCD}$ ). In the present study we investigated the victims who had previously recorded ECG available $(n=1,100,19 \%)$. Sixty-three percent $(n=689)$ of these had ischemic heart disease as a cause of SCD whereas 37\% $(\mathrm{n}=411)$ had non-ischemic heart disease as a cause of SCD. Collection of the study subjects is presented in Figure 1.

Determination of the underlying heart disease was based on medical records, questionnaires to the relatives and autopsy data. The death was defined as ischemic if there was evidence of coronary artery disease $(\mathrm{CAD})$ and a fresh intracoronary thrombus, plaque rupture or erosion, intraplaque hemorrhage, or critical coronary stenosis (>50\%) in major coronary vessel/branch. The cases with no evidence of relevant CAD were classified as non-ischemic and further classified based on ICD-10 classes. The classification in 
Figure 2 was used for more detailed descriptions of the heart disease based on postmortem findings, medical records and specific questionnaires of relatives. Criteria for each diagnosis have been described earlier ${ }^{3}$. Autopsy procedures included meticulous gross cardiac examinations, including visual and microscopic (selected cases) coronary artery examination, valve investigation, heart weight measurement and determination of myocardial fibrosis based on macroscopic and histological investigation on tissue samples taken from the heart. Histological examination was performed in all cases and a toxicology investigation was performed when autopsy findings were insufficient to define a cause of death, or if there was any suspicion of extensive alcohol intake or medication or drug use. Quantification of myocardial fibrosis was categorized into four groups; 1) no fibrosis, 2) scattered mild fibrosis, 3) moderate patchy fibrosis and 4) substantial fibrosis. The four-degree fibrosis classification is based on forensic pathologist's verbal statement of the amount of myocardial fibrosis in the heart. The statement was based on comprehensive evaluation of myocardial fibrosis in macroscopic myocardial dissection and 3-5 histological tissue samples from different myocardial sites.

\section{Electrocardiography}

All ECGs were recorded in a supine position at a paper speed of $50 \mathrm{~mm} / \mathrm{s}$ and a calibration of $1 \mathrm{mV} / 10$ mm. If multiple ECGs were available, the most recent one was used. ECGs were analyzed independently by 2 researchers. Median time from ECG recording to SCD was 2 years (interquartile range 0.28 to 4.9 years, maximum time 34 years, 92\% $\leq 10$ years, $82 \% \leq 5$ years). QRS and JT durations were calculated from lead $\mathrm{V}_{5}$. Intraventricular conduction delay (IVCD) was defined as QRS duration $>110 \mathrm{~ms}$ in the absence of bundle branch blocks (BBB) meeting standard criteria ${ }^{8}$. JT interval was corrected for heart rate by the Bazzett formula. Sokolow-Lyon voltage index was calculated as the sum of $\mathrm{SV}_{1}$ and $\mathrm{RV}_{5}$ or $\mathrm{RV}_{6}$ based upon the highest R-wave amplitude. QRS fragmentation and inferolateral early repolarization (ER) were defined according to previous literature ${ }^{9,10}$ and T-wave inversions were defined as T-wave amplitude $\leq-0.1 \mathrm{mV}$ in at least two anterior $\left(\mathrm{V}_{1}-\mathrm{V}_{3}\right)$, lateral $\left(\mathrm{I}, \mathrm{aVL}, \mathrm{V}_{4}-\mathrm{V}_{6}\right)$ and/or inferior (II, aVF, III) leads. Pathologic Q waves were defined as Q waves in at least 2 continuous leads meeting following criteria; Q 
waves (or absent $R$ waves) greater than $20 \mathrm{~ms}$ in duration in leads $\mathrm{V}_{2}$ and $\mathrm{V}_{3}, \geq 40 \mathrm{~ms}$ and at least $0.1 \mathrm{mV}$ deep in lead III and $\geq 30 \mathrm{~ms}$ and deeper than $0.1 \mathrm{mV}$ in other leads. ECG was stated as normal in the absence of QRS fragmentation, pathologic Q waves, prolonged QRS complex (>110ms), T-wave inversions, positive T waves in aVR lead, prolonged QTc interval (440ms in women, 460ms in men), inferolateral early repolarization and Sokolow-Lyon index $>3.5 \mathrm{mV}$.

\section{Statistical methods}

Continuous variables are expressed as mean \pm SD. For the continuous variables, the data was assumed to be sufficiently normally distributed if the skewness was between -1 and 1 . If skewed distribution (|skewness $\mid>1$, QRS duration, BMI) was encountered, the variable was transformed into natural logarithm. Distributions were thereafter verified as symmetric. For continuous QRS duration, p-values are calculated from natural logarithm whereas unstandardized $\beta$ values and confidence intervals were calculated from absolute values. Between-groups differences were analyzed by $\chi^{2}$-test for categorical and two-sided t-test or analysis of variance (ANOVA) for continuous variables. In case of significance main effect between multiple groups, post hoc analysis by $\chi^{2}$ and Bonferroni test were applied for categorical and continuous variables, respectively. Linear and logistic regression models were used to assess the effect of fibrosis on ECG variables independently from heart weight. In table 2 we evaluated usefulness of different ECG parameters in assessing the possibility of at least moderate amount of fibrosis. All analyses were performed with the Statistical Package for Social Studies version 21.0 (SPSS Inc, Chicago, IL). All $\mathrm{p}$ values are 2 -sided and values less than 0.05 were considered as statistically significant.

This research was done without public involvement. Patients were not involved in the design, or conduct, or reporting, or dissemination plans of our research. The study complies with the Declaration of Helsinki, and the Ethics Committee of the Oulu University Hospital approved the study (Decision number 21/2007). A permit to use data from medico-legal death investigations was obtained from the National Institute for Health and Welfare and the Regional State Administrative Agency of Northern Finland. 


\section{RESULTS}

\section{Characteristics}

Some degree of myocardial fibrosis was present in $91 \%$ of the ischemic and $93 \%$ of the non-ischemic SCD victims. Seventeen subjects $(1.5 \%)$ had ECG taken for reasons that could have affect the ECG measurements, such as ischemia. The mean age of the study subjects was $66 \pm 13$ years and $75 \%$ were male. Non-ischemic SCD victims were younger with mean age of $59 \pm 12$ years, compared to $70 \pm 11$ years among ischemic SCD victims ( $\mathrm{p}<0.001)$. Forty-eight percent of the non-ischemic SCD victims and $63 \%$ of the ischemic SCD victims had previously diagnosed cardiovascular disease. Mean heart weight at autopsy was $497 \pm 145 \mathrm{~g}$ among non-ischemic and $474 \pm 132 \mathrm{~g}$ among ischemic SCD victims. Abnormal ECGs were present in $75.3 \%$ of the subjects with no fibrosis, $73.7 \%$ in subjects with scattered mild fibrosis, $88.5 \%$ in subjects with moderate patchy fibrosis, and $91.7 \%$ in subjects with substantial fibrosis ( $p<0.001)$. Characteristics of the subjects are represented in Table 1.

\section{Depolarization abnormalities}

Myocardial fibrosis was associated with various depolarization abnormalities. QRS fragmentation and pathologic Q waves were more common, and QRS complexes wider, in association with the proportion of increasing myocardial fibrosis. Mean QRS duration was $96 \pm 21 \mathrm{~ms}$ among subjects with no fibrosis, $97 \pm 20 \mathrm{~ms}$ in subjects with mild fibrosis, $103 \pm 26 \mathrm{~ms}$ in subjects with moderate fibrosis, and $108 \pm 27 \mathrm{~ms}$ among subjects with substantial fibrosis $(\mathrm{p}<0.001, \beta=0.153)$. The prevalence of LBBB increased significantly in association with fibrosis $(\mathrm{p}=0.014)$. The incidence of IVCD also tended to increase in association with the severity of fibrosis, although the association did not reach statistical significance $(p=0.11)$. The prevalence of RBBB varied significantly between fibrosis groups $(p=0.050)$, although changes in prevalence were not linear. The prevalence of pathologic Q waves and fragmented QRS complex increased gradually, concomitant with the degree of fibrosis ( $\mathrm{p}<0.001$ for both). The presence of inferolateral ER had no significant association with the amount of fibrosis. Sokolow-Lyon index had 
statistically significant variability between the fibrosis groups, but the association was not linear and was not present in subgroup analyses.

\section{Repolarization abnormalities}

In addition to depolarization abnormalities, increasing myocardial fibrosis was also associated with Twave inversions $(\mathrm{p}<0.001)$ and the T-wave pattern in lead aVR. The T-wave in aVR tended to shift toward positive as the amount of fibrosis increased and positive T-waves in aVR were more common in groups with greater degrees of fibrosis $(\mathrm{p}<0.001)$. The mean T-wave amplitude was $-0.14 \pm 0.12 \mathrm{mV}$ in subjects without fibrosis, $-0.12 \pm 0.11 \mathrm{mV}$ in subjects with mild fibrosis, $-0.10 \pm 0.12 \mathrm{mV}$ in subjects with moderate fibrosis and $-.05 \pm 0.12 \mathrm{mV}$ in subjects with substantial fibrosis $(\mathrm{p}<0.001 ; \beta=0.18)$. Heart rate correlated JT interval was not affected by the degree of fibrosis. ECG measurements associated with the degree of fibrosis are provided in Table 1.

\section{Subgroup ECG analyses for ischemic and non-ischemic SCD victims}

Prolonged QRS duration and QRS fragmentation had statistically significant associations with the degree of fibrosis in both ischemic and non-ischemic subgroups. Trends of increasing $\mathrm{T}$ wave amplitude in aVR and prevalence of T-wave inversions and pathologic $\mathrm{Q}$ waves were present among both ischemic and non-ischemic SCD victims, but reached statistical significance only in ischemic SCD subgroup. The prevalence of positive $\mathrm{T}$ waves in aVR was $17.3 \%$ in ischemic SCD victims and $14.6 \%$ in non-ischemic SCD victims. ECG measurements specified according to the amount of fibrosis and ischemic versus nonischemic causes of SCD are presented in Figure 3. More detailed statistical analyses and characteristics for ischemic and non-ischemic SCD victims are presented in Supplementary material.

\section{Regression analysis}

Heart weight correlated positively with the degree of fibrosis $(\beta=0.25 ; \mathrm{p}<0.001)$. Independent of heart weight, QRS duration ( $\mathrm{p}=0.004$, unstandardized $\beta 2.1 \mathrm{~ms}, 95 \%$ CI: $0.5-3.8 \mathrm{~ms}$ per category on average), T-wave amplitude in aVR $(\mathrm{p}<0.001$, unstandardized $\beta 0.02 \mathrm{mV}, 95 \% \mathrm{CI}: 0.01-0.03 \mathrm{mV}$ per category on 
average), T-wave inversions (OR 1.5, 95\% CI 1.2-1.8 per group increase in fibrosis; $\mathrm{p}<0.001$ ), QRS fragmentation (OR 1.5, 95\% CI 1.2-1.7; $\mathrm{p}<0.001)$ and pathologic Q waves (OR 1.8, 95\% CI 1.4-2.3; $\mathrm{p}<0.001)$ retained independent association with the degree of fibrosis.

\section{ECG abnormalities in moderate-to-substantial fibrosis}

An additional analysis to assess the ability of ECGs to detect fibrotic accumulation was performed between cases with no/mild fibrosis and those with moderate or substantial fibrosis, since the prevalence of ECG abnormalities increased notably as the amount of myocardial fibrosis shifted from scattered mildto-moderate patchy fibrosis. All ECG measurements which had significant results in analyses of the 4 fibrosis groups were significantly associated in the analyses of the 2 combined groups. In addition, IVCD achieved significant association in the 2 combined group analyses. At least one ECG abnormality was present in $89.2 \%$ of those with moderate/substantial fibrosis as compared to $74.0 \%$ of those with no/mild fibrosis. The best sensitivity for detecting moderate/substantial fibrosis was in fragmented QRS complex (60.9\%) whereas LBBB had the best specificity (96.4\%). ECG characteristics between no/mild fibrosis and moderate/substantial fibrosis are represented in Table 2.

\section{DISCUSSION}

In this study, consisting of 1,100 autopsy-verified SCD victims with previously recorded ECG, we observed that increasing QRS duration, QRS fragmentation, pathologic Q waves, T-wave inversions and rising T-wave in the aVR lead were associated with increasing degrees of myocardial fibrosis in histological samples. Increased QRS complex durations were partially a result of LBBBs, but the prevalence of IVCD also tended to increase along with fibrosis. Although the amount of myocardial fibrosis correlated positively with heart weight, ECG abnormalities remained associated with myocardial fibrosis independently of hypertrophy based upon heart weight. The effect of fibrosis on depolarization abnormalities was observed in both ischemic and non-ischemic SCD victims, but repolarization 
abnormalities had no statistically significant correlations with fibrosis among non-ischemic SCD victims, although trends were observed.

An analysis comparing absence of fibrosis or mild fibrosis with moderate-to-substantial fibrosis demonstrated that fragmented QRS complex had the best sensitivity (60.9\%) and LBBB had the best specificity (96.4\%) for detecting moderate patchy or substantial amount of myocardial fibrosis. Greater than $25 \%$ of the subjects with scattered mild fibrosis at autopsy had normal ECG. This suggests that smaller amounts of myocardial fibrosis are more difficult to identify based on ECG findings, even though the risk for arrhythmia may be increased. Single ECG characteristics had a weaker ability to detect greater amounts of fibrosis, and the presence of multiple ECG abnormalities was notably better, since 9 out of 10 subjects with moderate-to-substantial fibrosis had abnormal ECGs.

Attenuated association between myocardial fibrosis and ECG abnormalities among non-ischemic SCD victims is likely due to the lower number of non-ischemic SCD victims compared to ischemic SCD victims, resulting in lower power of the statistical analyses. Effect of divergent fibrosis texture between ischemic and non-ischemic heart disease is not however excluded as fibrosis in ischemic heart disease is often result of local myocardial ischemia/infarction and subsequent scar formation, whereas non-ischemic heart disease tends to express more diffuse cardiomyocyte loss and fibrotic replacement ${ }^{11}$. Nevertheless, even if myocardial fibrosis did not correlate with ECG abnormalities as strongly among non-ischemic SCD victims, the prevalence of fibrosis-related depolarization and repolarization abnormalities were notably higher than in general population. In Finnish cohort of more than 10,000 participants, the prevalence of IVCD and LBBB were $0.6 \%$ and $0.3 \%$ respectively ${ }^{12}$. Among non-ischemic SCD victims, the corresponding percentages were $9.5 \%$ for IVCD and 5.8\% for LBBB, yielding a 16- and 19- times higher prevalence compared to general population. The prevalence of $\mathrm{T}$-wave inversions and positive $\mathrm{T}$ waves in aVR in the Finnish general population were $1.2 \%{ }^{13}$ and $2.2 \%,{ }^{14}$ respectively, indicating 15 - and 6.6 -times higher prevalence among non-ischemic SCD victims. 
In long-term follow-up and case-control studies, the same depolarization and repolarization abnormalities have been associated with increased risk of arrhythmic death in general population ${ }^{12-16}$. Arrhythmogenic properties of myocardial fibrosis have been demonstrated previously both in vitro ${ }^{17}$ and in clinical studies, in which fibrosis detected in MRI associated with increased risk of arrhythmic events ${ }^{18}$. The results of our study provide a likely explanation for the increased risk of SCD related to depolarization and repolarization abnormalities, in that they may represent an underlying fibrotic cardiac disease.

Electrophysiologic abnormalities may also have arrhythmogenic properties independently from structural defects, as in the case of long QT syndrome. In our previous studies we have reported inferolateral ER as a risk marker for both ischemic and non-ischemic $\mathrm{SCD}^{19,20}$. In this study, the prevalence of inferolateral ER did not correlate with myocardial fibrosis suggesting that ER may also represent independent electrical instability.

Parallel with our study, previous studies have also demonstrated that myocardial fibrosis detected as late gadolinium enhancement (LGE) in MRI correlates with fragmented QRS complex, ECG strain and Twave inversions ${ }^{21-25}$. The association between LGE and pathologic Q waves in hypertrophic cardiomyopathy patients has not been as congruent in previous studies ${ }^{25,26}$. However, LGE can detect only regional fibrosis patterns and ECG characteristics of diffuse fibrosis are less well studied.

Although current SCD prediction is insufficient and additional risk markers are being sought in current research, ECG-based SCD risk stratification have not proven to be useful enough to change clinical practice. The role of the ECG in SCD prediction and prevention resides primarily in the early recognition of subjects with life-threatening cardiac disease. In non-ischemic SCD victims, the death is often the first symptom of cardiac disease, since the disease is initially diagnosed at autopsy in more than one-half of these cases. ${ }^{3}$ In ischemic heart disease, previously asymptomatic fibrotic accumulation may be a result of previous silent myocardial infarction ${ }^{27}$. Although the likelihood of prior diagnosis increases in parallel with disease severity at autopsy, $28 \%$ of our study subjects with substantial fibrosis had no previous cardiovascular disease diagnosis. Treatment of the underlying cardiomyopathy is the cornerstone for SCD 
prevention as improved medical therapy and reduced sudden death rates in heart failure has narrowed the usefulness of implantable cardioverter defibrillator, especially in non-ischemic cardiac disease ${ }^{28,29}$. Meticulous investigations with cardiovascular imaging are reasonable, especially for patients with risk factors for life-threatening cardiomyopathies (e.g. obesity, hypertension, excessive alcohol consumption, family history of SCD) and myocardial fibrosis-related ECG abnormalities.

\section{Limitations}

One limitation of our study is the lack of a uniform quantitative histological classification of myocardial fibrosis, since the groups were based on subjective evaluation of fibrosis. However, autopsies were performed by highly experienced forensic pathologies since every unexpected SCD undergoes medicolegal autopsy and meticulous cardiac investigations in Finland. Consequently, the number of prior ECGs among autopsy-verified SCD victims is relatively high although we were not able to collect ECGs from all 5,869 SCD victims. Additionally, we do not have information regarding the region or transmural distribution of fibrosis pattern. Median time from ECG recording to SCD was 2 years which can be probable source of bias. Nonetheless, the absolute number of ECGs might not result in enough power for valid subgroup analyses in this study.

\section{Conclusions}

QRS prolongation, pathologic Q waves, fragmented QRS complex, T-wave inversions and positive Twaves in aVR lead were prevalent and associated with fibrotic accumulation in the myocardium among SCD victims. The results provide a likely explanation for the increased SCD risk related to abnormal QRS complex and inverted T waves. Repolarization abnormalities had stronger associations with fibrosis among ischemic SCD victims. Patients with such ECG abnormalities without known cardiac disease and risk factors for ischemic and non-ischemic cardiomyopathies require further investigations for diagnosis and classification that could possibly affect the identification risk for SCD in smaller subgroups and individuals. 


\section{ACKNOWLEDGEMENTS}

This work was performed in the Institute of Clinical Medicine, University of Oulu, Oulu, Finland. The authors acknowledge all the help with data gathering by the Oulu University Hospital staff.

\section{FUNDING}

The study was supported by Sigrid Juselius Foundation, Finnish Foundation for Cardiovascular Research, Yrjö Jahnsson Foundation, Paavo Nurmi Foundation, Paulo Foundation, Aarne Koskelo Foundation, Finnish Medical Foundation, Aatos and Jane Erkko Foundation.

\section{CONTRIBUTORS}

Study design and conception: Myerburg, Huikuri, Junttila. Acquisition, analysis, or interpretation of data: Holmström, Haukilahti, Vähätalo, Kenttä, Appel, Pakanen, Myerburg, Huikuri, Junttila. Drafting of the manuscript: Holmström, Junttila. Statistical analysis: Holmström, Haukilahti, Kenttä, Kiviniemi. Critical revision of the manuscript for important intellectual content: Haukilahti, Vähätalo, Kenttä, Pakanen, Myerburg, Huikuri, Junttila. Obtained funding: Junttila. Supervision: Huikuri, Junttila. Guarantors: Holmström, Junttila.

\section{COMPETING INTERESTS}

No conflicts of interest.

The Corresponding Author has the right to grant on behalf of all authors and does grant on behalf of all authors, an exclusive licence (or non exclusive for government employees) on a worldwide basis to the BMJ Publishing Group Ltd and its Licensees to permit this article (if accepted) to be published in HEART editions and any other BMJPGL products to exploit all subsidiary rights.

\section{REFERENCES}


1. Junttila MJ, Hookana E, Kaikkonen KS, et al. Temporal trends in the clinical and pathological characteristics of victims of sudden cardiac death in the absence of previously identified heart disease. Circ Arrhythm Electrophysiol. 2016;9:10.1161/CIRCEP.115.003723.

2. Myerburg RJ, Junttila MJ. Sudden cardiac death caused by coronary heart disease. Circulation. 2012;125:1043-1052.

3. Hookana E, Junttila MJ, Puurunen VP, et al. Causes of nonischemic sudden cardiac death in the current era. Heart Rhythm. 2011;8:1570-1575.

4. Stecker EC, Vickers C, Waltz J, et al. Population-based analysis of sudden cardiac death with and without left ventricular systolic dysfunction: Two-year findings from the oregon sudden unexpected death study. J Am Coll Cardiol. 2006;47:1161-1166.

5. Myerburg RJ, Goldberger JJ. Sudden cardiac arrest risk assessment: Population science and the individual risk mandate. JAMA Cardiol. 2017;2:689-694.

6. Narayanan K, Chugh SS. The 12-lead electrocardiogram and risk of sudden death: Current utility and future prospects. Europace. 2015;17(Suppl 2):ii7-13.

7. Lahti RA. From findings to statistics: An assessment of Finnish medical cause-of-death information in relation to underlying-cause coding. Helsinki, Finland: University Printing House 2005.

8. Surawicz B, Childers R, Deal BJ, et al. AHA/ACCF/HRS recommendations for the standardization and interpretation of the electrocardiogram: Part III: Intraventricular conduction disturbances: A scientific statement from the american heart association electrocardiography and arrhythmias committee, council on clinical cardiology; the american college of cardiology foundation; and the heart rhythm society. endorsed by the international society for computerized electrocardiology. J Am Coll Cardiol. 2009;53:976-981. 
9. Das MK, Khan B, Jacob S, et al. Significance of a fragmented QRS complex versus a $Q$ wave in patients with coronary artery disease. Circulation. 2006;113:2495-2501.

10. Tikkanen JT, Anttonen O, Junttila MJ, et al. Long-term outcome associated with early repolarization on electrocardiography. N Engl J Med. 2009;361:2529-2537.

11. Jellis C, Martin J, Narula J, et al. Assessment of nonischemic myocardial fibrosis. J Am Coll Cardiol. 2010;56:89-97.

12. Aro AL, Anttonen O, Tikkanen JT, et al. Intraventricular conduction delay in a standard 12-lead electrocardiogram as a predictor of mortality in the general population. Circ Arrhythm Electrophysiol. 2011;4:704-710.

13. Aro AL, Anttonen O, Tikkanen JT, et al. Prevalence and prognostic significance of T-wave inversions in right precordial leads of a 12-lead electrocardiogram in the middle-aged subjects. Circulation. $2012 ; 125: 2572-2577$.

14. Anttila I, Nikus K, Nieminen T, et al. Relation of positive T wave in lead aVR to risk of cardiovascular mortality. Am J Cardiol. 2011;108:1735-1740.

15. Terho HK, Tikkanen JT, Junttila JM, et al. Prevalence and prognostic significance of fragmented QRS complex in middle-aged subjects with and without clinical or electrocardiographic evidence of cardiac disease. Am J Cardiol. 2014;114:141-147.

16. Toukola T, Junttila MJ, Holmstrom LTA, et al. Fragmented QRS complex as a predictor of exerciserelated sudden cardiac death. J Cardiovasc Electrophysiol. 2018;29:55-60.

17. Nguyen TP, Qu Z, Weiss JN. Cardiac fibrosis and arrhythmogenesis: The road to repair is paved with perils. J Mol Cell Cardiol. 2014;70:83-91. 
18. Ganesan AN, Gunton J, Nucifora G, et al. Impact of late gadolinium enhancement on mortality, sudden death and major adverse cardiovascular events in ischemic and nonischemic cardiomyopathy: A systematic review and meta-analysis. Int J Cardiol. 2018;254:230-237.

19. Holmstrom LTA, Haukilahti MA, Tikkanen JT, et al. Inferolateral early repolarization among nonischaemic sudden cardiac death victims. Europace. 2018;20(FI1):f93-f98.

20. Tikkanen JT, Wichmann V, Junttila MJ, et al. Association of early repolarization and sudden cardiac death during an acute coronary event. Circ Arrhythm electrophysiol. 2012;5:714-718.

21. Park $\mathrm{CH}$, Chung H, Kim Y, et al. Electrocardiography based prediction of hypertrophy pattern and fibrosis amount in hypertrophic cardiomyopathy: Comparative study with cardiac magnetic resonance imaging. Int J Cardiovasc Imaging. 2018;34:1619-1628.

22. Konno T, Hayashi K, Fujino N, et al. Electrocardiographic QRS fragmentation as a marker for myocardial fibrosis in hypertrophic cardiomyopathy. J Cardiovasc Electrophysiol. 2015;26:1081-1087.

23. Shah AS, Chin CW, Vassiliou V, et al. Left ventricular hypertrophy with strain and aortic stenosis. Circulation. 2014;130:1607-1616.

24. Chen X, Zhao S, Zhao T, et al. T-wave inversions related to left ventricular basal hypertrophy and myocardial fibrosis in non-apical hypertrophic cardiomyopathy: A cardiovascular magnetic resonance imaging study. Eur J Radiol. 2014;83:297-302.

25. Fronza M, Raineri C, Valentini A, et al. Relationship between electrocardiographic findings and cardiac magnetic resonance phenotypes in patients with hypertrophic cardiomyopathy. Int J Cardiol Heart Vasc. 2016;11:7-11. 
26. Chen X, Zhao T, Lu M, et al. The relationship between electrocardiographic changes and CMR features in asymptomatic or mildly symptomatic patients with hypertrophic cardiomyopathy. Int $J$ Cardiovasc Imaging. 2014;30(Suppl 1):55-63.

27. Vahatalo JH, Huikuri HV, Holmstrom LTA, et al. Association of silent myocardial infarction and sudden cardiac death. JAMA Cardiol. 2019;4:796-802.

28. Shen L, Jhund PS, Petrie MC, et al. Declining risk of sudden death in heart failure. $N$ Engl J Med. 2017;377:41-51.

29. Kober L, Thune JJ, Nielsen JC, et al. Defibrillator implantation in patients with nonischemic systolic heart failure. N Engl J Med. 2016;375:1221-1230. 


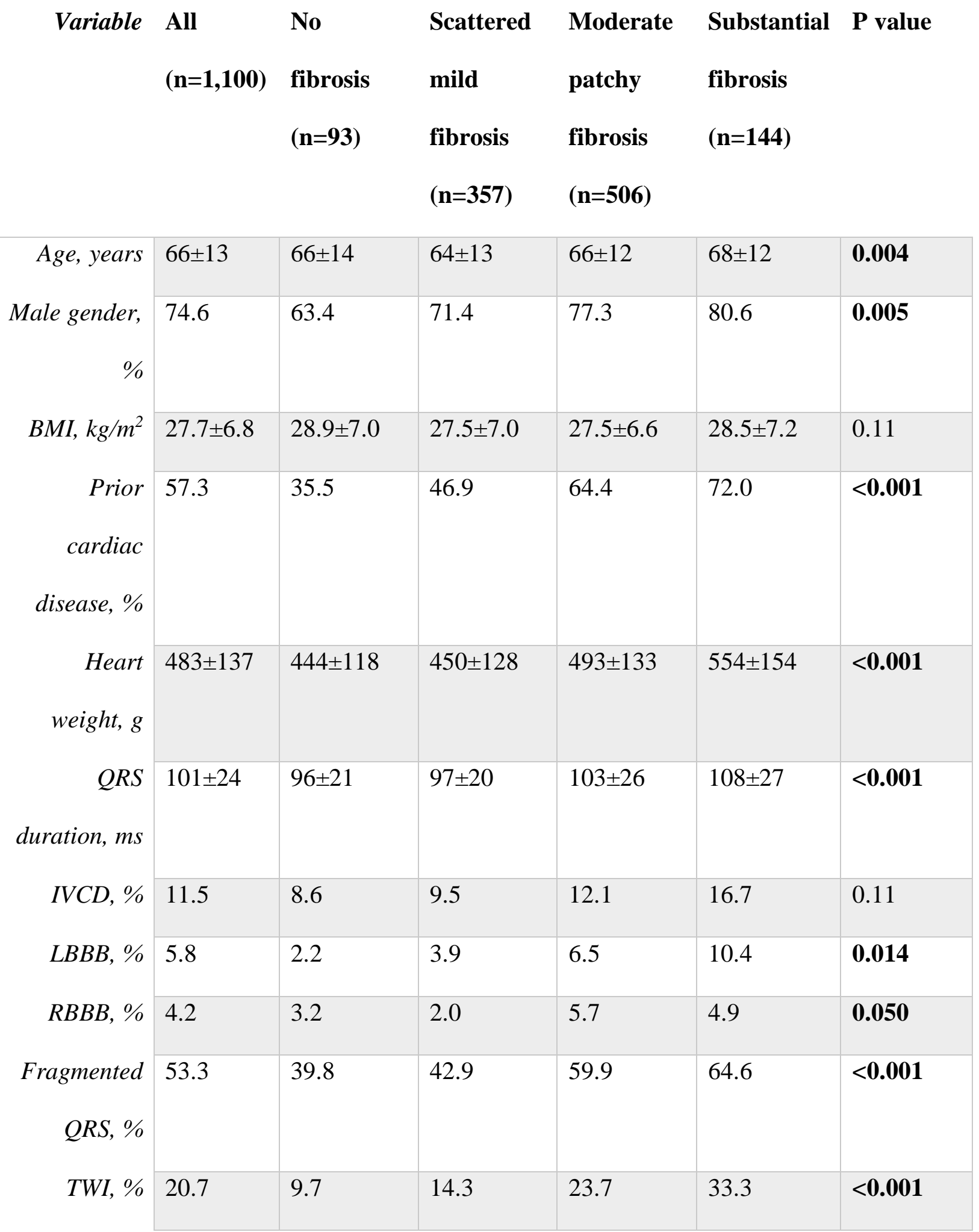




\begin{tabular}{|c|c|c|c|c|c|c|}
\hline $\begin{array}{l}\text { T-wave } \\
\text { amplitude } \\
\text { (aVR), } m V\end{array}$ & $\begin{array}{l}- \\
0.10 \pm 0.12\end{array}$ & $-0.14 \pm 0.12$ & $-0.12 \pm 0.11$ & $-0.10 \pm 0.12$ & $-0.05 \pm 0.12$ & $<0.001$ \\
\hline$a V R T+, \%$ & 16.3 & 9.7 & 11.8 & 17.4 & 27.8 & $<0.001$ \\
\hline $\begin{array}{r}\text { Inferolateral } \\
E R, \%\end{array}$ & 21.0 & 16.1 & 20.7 & 21.7 & 22.2 & 0.65 \\
\hline $\begin{array}{r}\text { Pathologic } Q \\
\text { waves, } \%\end{array}$ & 13.7 & 4.3 & 9.2 & 14.6 & 27.8 & $<0.001$ \\
\hline$J T c, m s$ & $327 \pm 36$ & $325 \pm 36$ & $326 \pm 32$ & $329 \pm 37$ & $321 \pm 42$ & 0.19 \\
\hline $\begin{array}{r}\text { Sokolow- } \\
\text { Lyon index, } \\
m V\end{array}$ & $1.9 \pm 1.0$ & $1.8 \pm 0.9$ & $1.8 \pm 0.9$ & $2.0 \pm 1.0$ & $1.9 \pm 1.0$ & 0.004 \\
\hline $\begin{array}{r}\text { Abnormal } \\
\text { ECG, \% }\end{array}$ & 83.0 & 75.3 & 73.7 & 88.5 & 91.7 & $<0.001$ \\
\hline
\end{tabular}

Table 1. Characteristics and ECG findings classified by the degree of fibrosis among all SCD victims with prior ECG $(n=1,100)$. aVRT+= positive $\mathrm{T}$ wave in lead aVR, ER=Early repolarization, IVCD=Intraventricular conduction delay, $\mathrm{LBBB}=$ Left bundle branch block, NS=Not significant, RBBB=Right bundle branch block, TWI=T-wave inversion. ECG was stated as normal in the absence of QRS fragmentation, pathologic Q waves, prolonged QRS complex (>110ms), T-wave inversions, positive T waves in aVR lead, prolonged QTc interval (440ms in women, 460ms in men), inferolateral early repolarization and Sokolow-Lyon index $>3.5 \mathrm{mV}$. 


\section{fibrosis $(n=450) \quad$ patchy/substantial}

fibrosis $(\mathbf{n}=650)$

\begin{tabular}{|r|l|l|l|}
\hline QRS $>110 \mathrm{~ms}, \%$ & 14.4 & 26.8 & $<0.001$ \\
\hline LBBB, \% & 3.6 & 7.4 & 0.008 \\
\hline IVCD, \% & 9.3 & 13.1 & 0.056 \\
\hline Fragmented QRS, & 42.2 & 60.9 & $<0.001$ \\
\hline Pathologic Q & 8.2 & & $<0.001$ \\
waves, \% & & 17.5 & \\
TWI, \% & 13.3 & & $<0.001$ \\
aVRT+, \% & 11.3 & 25.8 & $<0.001$ \\
Any ECG & 74.0 & 19.7 & $<0.001$ \\
abnormality, \% & & 89.2 & \\
\hline
\end{tabular}

Table 2. ECG abnormalities in subjects with at least moderate patchy fibrosis in comparison to those with no more than scattered mild fibrosis. aVRT $+=$ positive $\mathrm{T}$ wave in lead aVR, IVCD=Intraventricular conduction delay, $\mathrm{LBBB}=\mathrm{Left}$ bundle branch block, TWI=T-wave inversion. ECG was stated as normal in the absence of QRS fragmentation, pathologic Q waves, prolonged QRS complex (>110ms), T-wave inversions, positive T waves in aVR lead, prolonged QTc interval (440ms in women, 460ms in men), inferolateral early repolarization and Sokolow-Lyon index $>3.5 \mathrm{mV}$. 
